\title{
Theorists snap over string pieces
}

Two recently published books are riling the small but influential community of string theorists, by arguing that the field is wandering dangerously far from the mainstream.

The books' titles say it all: Not Even Wrong, a phrase that physicist Wolfgang Pauli used to describe incomplete ideas, and The Trouble with Physics: The Rise of String Theory, the Fall of a Science, and What Comes Next. Both articulate a fear that the field is becoming too abstract and is focusing on aesthetics rather than reality. Some physicists even warn that the theory's dominance could pose a threat to the scientific method itself (see page 507).

Those accusations are vehemently denied by string theorists, and the books - written by outsiders - have stirred deep resentment in the tight-knit community. Not Even Wrong was published in June and The Trouble with Physics came out in September; shortly after they appeared on the Amazon books website, string theorist Luboš Motl of Harvard University posted reviews furiously entitled "Bitter emotions and obsolete understanding of high-energy physics" and "Another postmodern diatribe against modern physics and scientific method". As Nature went to press, the reviews had been removed.

Few in the community are, at least publicly, as vitriolic as Motl. But many are angry and struggling to deal with the criticism. "Most of my friends are quietly upset," says Leonard Susskind, a string theorist at Stanford University in California.

String theory postulates that the Universe consists of tiny strings vibrating in ten or so dimensions. Its fortunes have been buoyed by popular books in the past - most notably

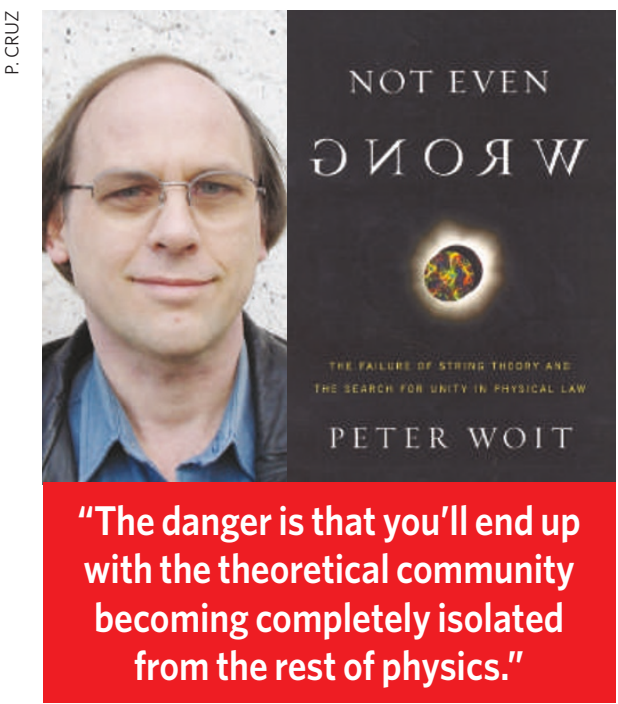

Brian Greene's 1999 bestseller The Elegant Universe, which said that the approach might unify the incompatible theories of gravity and quantum mechanics.

\section{Strung up}

But the theory has its share of problems, and these are the focus of the new works. For one thing, recent calculations suggest that it generates $10^{500}$ possible models of the Universe (see Nature 439, 10-12; 2006). This renders the theory essentially meaningless, according to critics. When these countless possibilities were first announced, Lee Smolin was already working on the book that eventually became The Trouble With Physics. A physicist at the Perimeter Institute for Theoretical Physics in Waterloo, Canada, Smolin had previously worked on string theory. "I and many other people began to get worried," he says.

Another difficulty is that if strings exist, they would be detectable only at energies far above anything that today's experiments can measure. "As you're not constrained by having to reproduce experiments, you can go off and play with whatever you want," says Peter Woit, a mathematician at Columbia University in New York City, and author of Not Even Wrong. "The danger is that you'll end up with the theoretical community becoming completely isolated from the rest of physics."

Smolin, whose book promotes an alternative theory known as loop quantum gravity, adds that string theorists have intentionally cut themselves off. "None of the major string theory groups has hired a postdoc or faculty member working in any of the other approaches to quantum gravity," he says. "But other research groups in quantum gravity have often hired young people working in string theory out of a sense that it should be encouraged."

\section{Boundary issues}

String theorists dispute the claim that they are isolating themselves. In recent years the theory has contributed significantly to heavyion physics, according to Joe Polchinski, a string theorist at the Kavli Institute for Theoretical Physics in Santa Barbara, California. When the Relativistic Heavy Ion Collider at Brookhaven National Laboratory in Upton, New York, first produced a hot quark gas, it was string theory that correctly predicted, retrospectively, some of the gas's properties. "In many ways, I feel the boundaries with other areas of physics are coming down," Polchinski says.

Warren Perkins, a cosmologist and particle

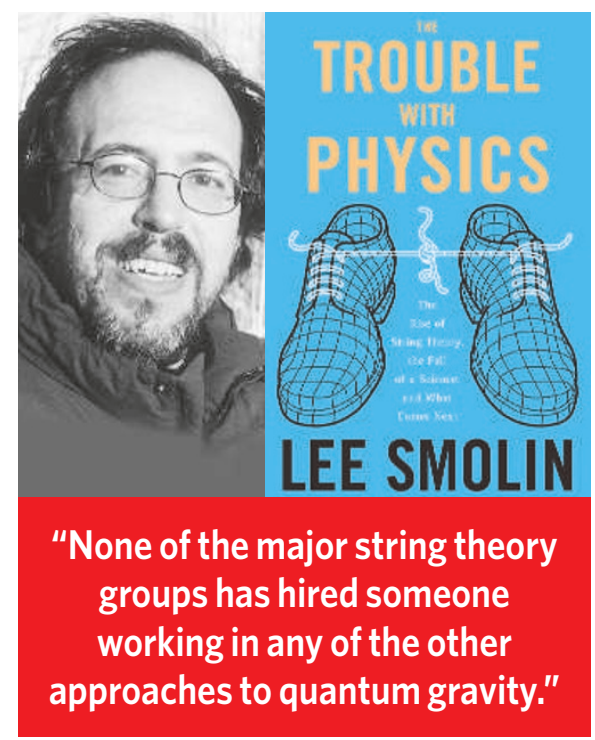

theorist at Swansea University in Wales, agrees. In recent years, he says, string theory has been proven equivalent to a conventional 'field theory', of the type being used to predict how particles will behave at the soon-to-open Large Hadron Collider, sited near Geneva. "There has been a lot of cross-talk between field theory and string theory," says Perkins. In some cases, string theory has provided far simpler approximations than its field-theory counterpart.

But strings have yet to provide the elusive link between gravity and quantum mechanics, hoped for by so many theorists. "The claims, when it comes to theoretical physics, tend to be exaggerated," says Abhay Ashtekar, who works on quantum gravity at Pennsylvania State University in University Park. He believes the inability of the community to live up to those expectations has made it defensive.

The books leave string theorists such as Susskind wondering how to approach such strong public criticism. "I don't know if the right thing is to worry about the public image or keep quiet," he says. He fears the argument may "fuel the discrediting of scientific expertise".

That's something that Smolin and Woit insist they don't want. Woit says his problem isn't with the theory itself, just some of its more grandiose claims. "There are some real things you can do with string theory," he says.

Smolin agrees, and says he hopes theorists will read his book to get a better understanding of his specific issues. "If they don't want to buy it, tell them to get in touch with me and I'll send them a copy."

Geoff Brumfiel 